\title{
ON THE DIOPHANTINE EQUATION $x_{1}^{6}+x_{2}^{6}+x_{3}^{6}=y_{1}^{6}+y_{2}^{6}+y_{3}^{6}$
}

\author{
JEAN-JOËL DELORME
}

\begin{abstract}
In this paper, we develop an elementary method for producing parametric solutions of the equation $x_{1}^{6}+x_{2}^{6}+x_{3}^{6}=y_{1}^{6}+y_{2}^{6}+y_{3}^{6}$ by reducing the resolution of a system including it to that of the equation

$$
\begin{aligned}
\left(s_{1}^{2}+\right. & \left.\left(s_{1}+t_{1}\right)^{2}\right)\left(s_{2}^{2}+\left(s_{2}+t_{2}\right)^{2}\right)\left(s_{3}^{2}+\left(s_{3}+t_{3}\right)^{2}\right) \\
& =\left(t_{1}^{2}+\left(s_{1}+t_{1}\right)^{2}\right)\left(t_{2}^{2}+\left(s_{2}+t_{2}\right)^{2}\right)\left(t_{3}^{2}+\left(s_{3}+t_{3}\right)^{2}\right) .
\end{aligned}
$$
\end{abstract}

We give such solutions of degrees $4,5,7,8,9$, and 11 .

\section{INTRODUCTION}

We say that a solution $\left(x_{1} ; x_{2} ; x_{3} ; y_{1} ; y_{2} ; y_{3}\right)$ of the equation $x_{1}^{6}+x_{2}^{6}+x_{3}^{6}=$ $y_{1}^{6}+y_{2}^{6}+y_{3}^{6}$, or of any system including it, is trivial if and only if each of the triples $\left(x_{1}^{2} ; x_{2}^{2} ; x_{3}^{2}\right)$ and $\left(y_{1}^{2} ; y_{2}^{2} ; y_{3}^{2}\right)$ may be deduced from the other by a permutation of its components. The first nontrivial solution, in positive integers, was given in 1934 by Subba-Rao [6], viz., $3^{6}+19^{6}+22^{6}=10^{6}+15^{6}+23^{6}$. It is "the smallest" nontrivial solution, in that $x_{1}^{6}+x_{2}^{6}+x_{3}^{6}$ is minimum. In 1967, Lander, Parkin, and Selfridge [5] gave ten nontrivial solutions in integers without any common factor. All these solutions, except one, satisfy the additional equality $x_{1}^{2}+x_{2}^{2}+x_{3}^{2}=y_{1}^{2}+y_{2}^{2}+y_{3}^{2}$. In 1970, Brudno [2] proved that the following system $\left(B_{1}\right)$ has an infinity of solutions:

$$
\left\{\begin{array}{l}
x_{1}^{6}+x_{2}^{6}+x_{3}^{6}=y_{1}^{6}+y_{2}^{6}+y_{3}^{6}, \\
x_{1}^{2}+x_{2}^{2}+x_{3}^{2}=y_{1}^{2}+y_{2}^{2}+y_{3}^{2}, \\
y_{2}=x_{2}-x_{3}, \\
y_{3}=x_{2}+x_{3} .
\end{array}\right.
$$

In 1974, Brudno and Kaplansky [3] obtained all the solutions of the preceding system. In 1976, Brudno [4] exhibited an infinity of solutions of the following system $\left(B_{2}\right)$ :

$$
\left\{\begin{array}{l}
x_{1}^{6}+x_{2}^{6}+x_{3}^{6}=y_{1}^{6}+y_{2}^{6}+y_{3}^{6}, \\
x_{1}^{2}+x_{2}^{2}+x_{3}^{2}=y_{1}^{2}+y_{2}^{2}+y_{3}^{2}, \\
3 x_{1}+x_{2}+x_{3}=3 y_{1}+y_{2}+y_{3},
\end{array}\right.
$$

in the form of homogeneous polynomial functions of degree four in two parameters. At the end of this paper, we mention important results obtained by

Received by the editor July 25, 1990 and, in revised form, June 28, 1991.

1991 Mathematics Subject Classification. Primary 11D41. 
A. Bremner in 1979. In the following, we shall exclusively consider solutions in rational numbers.

\section{Preliminaries}

We first reduce the resolution of a system including the equation $x_{1}^{6}+x_{2}^{6}+$ $x_{3}^{6}=y_{1}^{6}+y_{2}^{6}+y_{3}^{6}$ to that of a system of three quadratic equations.

Theorem 1. Consider the two systems:

$$
\begin{aligned}
& \left\{\begin{array}{l}
x_{1}^{6}+x_{2}^{6}+x_{3}^{6}=y_{1}^{6}+y_{2}^{6}+y_{3}^{6}, \\
x_{1}^{2}+x_{2}^{2}+x_{3}^{2}=y_{1}^{2}+y_{2}^{2}+y_{3}^{2}, \\
x_{1} y_{1}\left(x_{1}^{2}-y_{1}^{2}\right)+x_{2} y_{2}\left(x_{2}^{2}-y_{2}^{2}\right)+x_{3} y_{3}\left(x_{3}^{2}-y_{3}^{2}\right)=0,
\end{array}\right. \\
& \left\{\begin{array}{l}
x_{1}^{2}+x_{1} y_{1}-y_{1}^{2}+x_{2}^{2}-x_{2} y_{2}-y_{2}^{2}=0, \\
x_{2}^{2}+x_{2} y_{2}-y_{2}^{2}+x_{3}^{2}-x_{3} y_{3}-y_{3}^{2}=0, \\
x_{3}^{2}+x_{3} y_{3}-y_{3}^{2}+x_{1}^{2}-x_{1} y_{1}-y_{1}^{2}=0 .
\end{array}\right.
\end{aligned}
$$

Then the following hold:

(a) Every solution of (II) is a solution of (I).

(b) If $\left(x_{1} ; x_{2} ; x_{3} ; y_{1} ; y_{2} ; y_{3}\right)$ is a nontrivial solution of $(\mathrm{I})$, then $\left(x_{1} ; x_{2}\right.$; $\left.x_{3} ; y_{1} ; y_{2} ; y_{3}\right)$ or $\left(x_{1} ; x_{2} ; x_{3} ;-y_{1} ;-y_{2} ;-y_{3}\right)$ is a solution of (II).

Proof of Theorem 1. For $1 \leq i \leq 3$, put $x_{i}^{2}+x_{i} y_{i}-y_{i}^{2}=A_{i}$ and $-x_{i}^{2}+x_{i} y_{i}+y_{i}^{2}=$ $B_{i}$. It is easily verified that $A_{i}-B_{i}=2\left(x_{i}^{2}-y_{i}^{2}\right), A_{i}^{2}-B_{i}^{2}=4 x_{i} y_{i}\left(x_{i}^{2}-y_{i}^{2}\right)$, and $A_{i}^{3}-B_{i}^{3}=2\left(x_{i}^{6}-x_{i}^{6}\right)$, so that $(\mathrm{I})$ is equivalent to the following system:

$$
\left\{\begin{array}{l}
A_{1}+A_{2}+A_{3}=B_{1}+B_{2}+B_{3}, \\
A_{1}^{2}+A_{2}^{2}+A_{3}^{2}=B_{1}^{2}+B_{2}^{2}+B_{3}^{2}, \\
A_{1}^{3}+A_{2}^{3}+A_{3}^{3}=B_{1}^{3}+B_{2}^{3}+B_{3}^{3},
\end{array}\right.
$$

and (II) is equivalent to $\left\{A_{1}=B_{1} ; A_{2}=B_{2} ; A_{3}=B_{3}\right\}$. So every solution of (II) is obviously a solution of (I).

Further, if $\left(x_{1} ; x_{2} ; x_{3} ; y_{1} ; y_{2} ; y_{3}\right)$ is a nontrivial solution of $(\mathrm{I})$, we easily deduce from ( $\left.\mathrm{I}^{\mathrm{bis}}\right)$ that $\left(A_{1} ; A_{2} ; A_{3}\right)$ is equal to one of the six triples $\left(B_{1} ; B_{2} ; B_{3}\right),\left(B_{1} ; B_{3} ; B_{2}\right),\left(B_{2} ; B_{1} ; B_{3}\right),\left(B_{2} ; B_{3} ; B_{1}\right),\left(B_{3} ; B_{1} ; B_{2}\right)$, or $\left(B_{3} ; B_{2} ; B_{1}\right)$.

Now suppose that for some integer $i$ satisfying $1 \leq i \leq 3$, we have $A_{i}=B_{i}$. For instance, assume that $A_{1}=B_{1}$. We then have $x_{1}^{2}+x_{1} y_{1}-y_{1}^{2}=-x_{1}^{2}+$ $x_{1} y_{1}+y_{1}^{2}$, hence $x_{1}^{2}=y_{1}^{2}$. From the first two equations of $(\mathrm{I})$, we then deduce that $x_{2}^{6}+x_{3}^{6}=y_{2}^{6}+y_{3}^{6}$ and $x_{2}^{2}+x_{3}^{2}=y_{2}^{2}+y_{3}^{2}$, so that $\left(x_{2}^{2} ; x_{3}^{2}\right)=\left(y_{2}^{2} ; y_{3}^{2}\right)$ or $\left(x_{2}^{2} ; x_{3}^{2}\right)=\left(y_{3}^{2} ; y_{2}^{2}\right)$. Thus the solution $\left(x_{1} ; x_{2} ; x_{3} ; y_{1} ; y_{2} ; y_{3}\right)$ is trivial.

Finally, $\left(A_{1} ; A_{2} ; A_{3}\right)$ is necessarily equal either to $\left(B_{2} ; B_{3} ; B_{1}\right)$ or to $\left(B_{3}\right.$; $\left.B_{1} ; B_{2}\right)$, so that $\left(x_{1} ; x_{2} ; x_{3} ; y_{1} ; y_{2} ; y_{3}\right)$ or $\left(x_{1} ; x_{2} ; x_{3} ;-y_{1} ;-y_{2} ;-y_{3}\right)$ is a solution of (II).

\section{LINK MATRICES}

Notation. Let $\left(x_{1} ; x_{2} ; x_{3} ; y_{1} ; y_{2} ; y_{3}\right)$ be a sextuple of rational numbers. For $1 \leq i \leq 3$, put $V_{i}=\left(\begin{array}{c}x_{i} \\ y_{i}\end{array}\right)$. In the following, we shall write $\left(x_{1} ; x_{2} ; x_{3} ; y_{1} ; y_{2} ; y_{3}\right)$ or $\left(V_{1} ; V_{2} ; V_{3}\right)$ interchangeably. 
Using suitable matrices $M_{1}, M_{2}$, and $M_{3}$ such that $M_{1} V_{1}=V_{2}, M_{2} V_{2}=$ $V_{3}$, and $M_{3} V_{3}=V_{1}$, we reduce the resolution of system (II) to that of a single factorized equation.

Definition. 1. We call every 2 by 2 matrix of rational entries of the form $\left(\begin{array}{cc}a-b & a \\ b & a-b\end{array}\right)$ with determinant -1 a link matrix.

2. We call every triple of link matrices, $\left(M_{1} ; M_{2} ; M_{3}\right)$, such that the trace of the matrix $M_{3} M_{2} M_{1}$ is zero, a solving triple.

Theorem 2. (a) Let $\left(V_{1} ; V_{2} ; V_{3}\right)$ be a nonzero solution of (II). There exists a unique solving triple such that $M_{1} V_{1}=V_{2}, M_{2} V_{2}=V_{3}$, and $M_{3} V_{3}=V_{1}$.

(b) Let $\left(M_{1} ; M_{2} ; M_{3}\right)$ be a solving triple. There exists a nonzero solution of (II), ( $\left.V_{1} ; V_{2} ; V_{3}\right)$, unique apart from a nonzero rational factor, such that $M_{1} V_{1}=V_{1}, M_{2} V_{2}=V_{3}$, and $M_{3} V_{3}=V_{1}$.

Definition. We shall say that $\left(M_{1} ; M_{2} ; M_{3}\right)$ is the solving triple associated with the nonzero solution $\left(V_{1} ; V_{2} ; V_{3}\right)$ of (II), and that $\left(V_{1} ; V_{2} ; V_{3}\right)$ is an antecedent solution of the triple $\left(M ; M_{2} ; M_{3}\right)$.

Lemma. Let $\left(x_{1} ; x_{2} ; x_{3} ; y_{1} ; y_{2} ; y_{3} ;\right)$ be a nonzero solution of (II). For $(i, j)$ $\in\{(1 ; 2),(2 ; 3),(3 ; 1)\}$ we have:

(a) $\left(x_{i}^{2}+x_{j}^{2}\right)\left(x_{i}^{2}+x_{i} y_{i}-y_{i}^{2}\right)=\left(x_{i} y_{j}+y_{i} x_{j}\right)\left(x_{i} x_{j}+x_{i} y_{j}-y_{i} x_{j}\right)$,

(b) $\left(y_{i}^{2}+y_{j}^{2}\right)\left(x_{i}^{2}+x_{i} y_{i}-y_{i}^{2}\right)=\left(x_{i} y_{j}+y_{i} x_{j}\right)\left(y_{i} y_{j}+x_{i} y_{j}-y_{i} x_{j}\right)$,

(c) $\left(x_{i} ; y_{i}\right) \neq(0 ; 0), x_{i}^{2}+x_{i} y_{i}-y_{i}^{2} \neq 0, x_{i}^{2}+x_{j}^{2} \neq 0$, and $x_{i} y_{j}+y_{i} x_{j} \neq 0$.

Proof. (a) For any rational numbers $x_{1}, y_{1}, x_{2}$, and $y_{2}$, we have the identity

$$
\begin{gathered}
\left(x_{1}^{2}+x_{2}^{2}\right)\left(x_{1}^{2}+x_{1} y_{1}-y_{1}^{2}\right)-\left(x_{1} y_{2}+y_{1} x_{2}\right)\left(x_{1} x_{2}+x_{1} y_{2}-y_{1} x_{2}\right) \\
=x_{1}^{2}\left(x_{1}^{2}+x_{1} y_{1}-y_{1}^{2}+x_{2}^{2}-x_{2} y_{2}-y_{2}^{2}\right) .
\end{gathered}
$$

If $\left(x_{1} ; x_{2} ; x_{3} ; y_{1} ; y_{2} ; y_{3}\right)$ is a solution of (II), we have $x_{1}^{2}+x_{1} y_{1}-y_{1}^{2}+x_{2}^{2}-$ $x_{2} y_{2}-y_{2}^{2}=0$, from which the result follows, for $(i ; j)=(1 ; 2)$. The other two cases are obtained by cyclic permutations of the indices 1,2 , and 3 .

(b) Similar proof, with the help of the identity

$$
\begin{aligned}
& \left(y_{1}^{2}+y_{2}^{2}\right)\left(x_{1}^{2}+x_{1} y_{1}-y_{1}^{2}\right)-\left(x_{1} y_{2}+y_{1} x_{2}\right)\left(y_{1} y_{2}+x_{1} y_{2}-y_{1} x_{2}\right) \\
& =y_{1}^{2}\left(x_{1}^{2}+x_{1} y_{1}-y_{1}^{2}+x_{2}^{2}-x_{2} y_{2}-y_{2}^{2}\right) .
\end{aligned}
$$

(c) (i) If, for instance, $\left(x_{1} ; y_{1}\right)=(0 ; 0)$, the first equation of (II) implies that $x_{2}^{2}-x_{2} y_{2}-y_{2}^{2}=0$, so that $\left(x_{2} ; y_{2}\right)=(0 ; 0)$, and the third equation implies that $x_{3}^{2}+x_{3} y_{3}-y_{3}^{2}=0$, so that $\left(x_{3} ; y_{3}\right)=(0 ; 0)$. Thus, $\left(x_{1} ; x_{2} ; x_{3} ; y_{1} ; y_{2} ; y_{3}\right)$ would be the zero solution, contrary to our hypothesis.

(ii) If, for instance, $x_{1}^{2}+x_{1} y_{1}-y_{1}^{2}=0$, then $\left(x_{1} ; y_{1}\right)=(0 ; 0)$, which brings us back to (i).

(iii) If, for instance, $x_{1}^{2}+x_{2}^{2}=0$, then $x_{1}=0$ and $x_{2}=0$. The first equation of (II) implies that $y_{1}^{2}+y_{2}^{2}=0$, so that $y_{1}=0$ and $y_{2}=0$. Therefore, we have $\left(x_{1} ; y_{1}\right)=(0 ; 0)$, which brings us back to $(\mathrm{i})$.

(iv) By the identity proved in (a), since $x_{i}^{2}+x_{j}^{2} \neq 0$ and $x_{i}^{2}+x_{i} y_{i}-y_{i}^{2} \neq 0$, we have $x_{i} y_{j}+y_{i} x_{j} \neq 0$. 
Proof of Theorem 2. (a) Let $\left(V_{1} ; V_{2} ; V_{3}\right)$ be a nonzero solution of (II). Let us solve the system

$$
\left(\begin{array}{cc}
a_{1}-b_{1} & a_{1} \\
b_{1} & a_{1}-b_{1}
\end{array}\right)\left(\begin{array}{l}
x_{1} \\
y_{1}
\end{array}\right)=\left(\begin{array}{l}
x_{2} \\
y_{2}
\end{array}\right)
$$

for $a_{1}$ and $b_{1}$. It may be written as $\left(x_{1}+y_{1}\right) a_{1}-x_{1} b_{1}=x_{2}, y_{1} a_{1}+\left(x_{1}-y_{1}\right) b_{1}=$ $y_{2}$. Its determinant, $x_{1}^{2}+x_{1} y_{1}-y_{1}^{2}$, is nonzero, by (c) of the lemma. Therefore, it has a unique solution, given by

$$
a_{1}=\frac{x_{1} x_{2}+x_{1} y_{2}-y_{1} x_{2}}{x_{1}^{2}+x_{1} y_{1}-y_{1}^{2}}, \quad b_{1}=\frac{y_{1} y_{2}+x_{1} y_{2}-y_{1} x_{2}}{x_{1}^{2}+x_{1} y_{1}-y_{1}^{2}}
$$

By (a) and (b) of the lemma, we also have

$$
a_{1}=\frac{x_{1}^{2}+x_{2}^{2}}{x_{1} y_{2}+y_{1} x_{2}}, \quad b_{1}=\frac{y_{1}^{2}+y_{2}^{2}}{x_{1} y_{2}+y_{1} x_{2}} \text {. }
$$

Finally,

$$
a_{1}-b_{1}=\frac{x_{1}^{2}+x_{2}^{2}-y_{1}^{2}-y_{2}^{2}}{x_{1} y_{2}+y_{1} x_{2}}=\frac{x_{2} y_{2}-x_{1} y_{1}}{x_{1} y_{2}+y_{1} x_{2}},
$$

from the first equation of (II). Thus,

$$
M_{1}=\frac{1}{x_{1} y_{2}+y_{1} x_{2}}\left(\begin{array}{cc}
x_{2} y_{2}-x_{1} y_{1} & x_{1}^{2}+x_{2}^{2} \\
y_{1}^{2}+y_{2}^{2} & x_{2} y_{2}-x_{1} y_{1}
\end{array}\right) .
$$

It then follows from Lagrange's identity that $\operatorname{det}\left(M_{1}\right)=-1$. Analogous proofs hold for $M_{2}$ and $M_{3}$, by cyclic permutations of the indices 1, 2, and 3. So there exists a unique triple $\left(M_{1} ; M_{2} ; M_{3}\right)$ of link matrices such that $M_{1} V_{1}=V_{2}$, $M_{2} V_{2}=V_{3}$, and $M_{3} V_{3}=V_{1}$.

Now we prove that the trace of the matrix $M_{3} M_{2} M_{1}$ is zero. It is easily verified that for any elements of a commutative ring, $a_{1}, a_{2}, a_{3}, b_{1}, b_{2}$, and $b_{3}$, we have

$$
\begin{aligned}
& \operatorname{Tr}\left(\left(\begin{array}{cc}
a_{3}-b_{3} & a_{3} \\
b_{3} & a_{3}-b_{3}
\end{array}\right)\left(\begin{array}{cc}
a_{2}-b_{2} & a_{2} \\
b_{2} & a_{2}-b_{2}
\end{array}\right)\left(\begin{array}{cc}
a_{1}-b_{1} & a_{1} \\
b_{1} & a_{1}-b_{1}
\end{array}\right)\right) \\
& \quad=2\left(a_{1} a_{2} a_{3}-b_{1} b_{2} b_{3}\right) .
\end{aligned}
$$

It follows that $\operatorname{Tr}\left(M_{3} M_{2} M_{1}\right)=2 \frac{N}{D}$, with

$$
\begin{aligned}
& N=\left(x_{1}^{2}+x_{2}^{2}\right)\left(x_{2}^{2}+x_{3}^{2}\right)\left(x_{3}^{2}+x_{1}^{2}\right)-\left(y_{1}^{2}+y_{2}^{2}\right)\left(y_{2}^{2}+y_{3}^{2}\right)\left(y_{3}^{2}+y_{1}^{2}\right), \\
& D=\left(x_{1} y_{2}+y_{1} x_{2}\right)\left(x_{2} y_{3}+y_{2} x_{3}\right)\left(x_{3} y_{1}+y_{3} x_{1}\right) .
\end{aligned}
$$

We may write

$$
N=\frac{1}{3}\left(\left(x_{1}^{2}+x_{2}^{2}+x_{3}^{2}\right)^{3}-\left(x_{1}^{6}+x_{2}^{6}+x_{3}^{6}\right)-\left(y_{1}^{2}+y_{2}^{2}+y_{3}^{2}\right)^{3}+\left(y_{1}^{6}+y_{2}^{6}+y_{3}^{6}\right)\right) .
$$

Now every solution of (II) is, by Theorem 1, a solution of (I), so that $x_{1}^{6}+x_{2}^{6}+$ $x_{3}^{6}=y_{1}^{6}+y_{2}^{6}+y_{3}^{6}$ and $x_{1}^{2}+x_{2}^{2}+x_{3}^{2}=y_{1}^{2}+y_{2}^{2}+y_{3}^{2}$. It follows that $N=0$, so that $\operatorname{Tr}\left(M_{3} M_{2} M_{1}\right)=0$. Thus, $\left(M_{1} ; M_{2} ; M_{3}\right)$ is indeed a solving triple.

(b) Let $\left(M_{1} ; M_{2} ; M_{3}\right)$ be a solving triple. We have $\operatorname{det}\left(M_{3} M_{2} M_{1}\right)=-1$, because $\operatorname{det}\left(M_{i}\right)=-1$ for $1 \leq i \leq 3$, and $\operatorname{Tr}\left(M_{3} M_{2} M_{1}\right)=0$. Hence, the matrix $M_{3} M_{2} M_{1}$ has the eigenvalues 1 and -1 . Let $V_{1}$ be an eigenvector of $M_{3} M_{2} M_{1}$, with rational coordinates, associated with the eigenvalue 1 . Define $V_{2}$ and $V_{3}$ by $M_{1} V_{1}=V_{2}$ and $M_{2} V_{2}=V_{3}$. We have $M_{3} V_{3}=M_{3} M_{2} V_{2}=$ 
$M_{3} M_{2} M_{1} V_{1}=V_{1}$. We now prove that $\left(V_{1} ; V_{2} ; V_{3}\right)$ is a solution of (II). We have

$$
\left(\begin{array}{l}
x_{2} \\
y_{2}
\end{array}\right)=\left(\begin{array}{cc}
a_{1}-b_{1} & a_{1} \\
b_{1} & a_{1}-b_{1}
\end{array}\right)\left(\begin{array}{l}
x_{1} \\
y_{1}
\end{array}\right) \text {. }
$$

It follows that

$$
x_{2}^{2}-x_{2} y_{2}-y_{2}^{2}=\operatorname{det}\left(M_{1}\right)\left(x_{1}^{2}+x_{1} y_{1}-y_{1}^{2}\right) \text {. }
$$

Now $\operatorname{det}\left(M_{1}\right)=-1$. We obtain $x_{1}^{2}+x_{1} y_{1}-y_{1}^{2}+x_{2}^{2}-x_{2} y_{2}-y_{2}^{2}=0$, which is the first equality of (II). The other equalities are obtained by cyclic permutations of the indices 1,2 , and 3 . Therefore, $\left(V_{1}, V_{2}, V_{3}\right)$ is a solution of (II), nonzero, since $V_{1}$ is nonzero.

Remark. If $\left(M_{1} ; M_{2} ; M_{3}\right)$ is a solving triple, so is $\left(M_{1} ; M_{2} ;-M_{3}\right)$.

We say that two nonzero solutions of (II) are conjugate if and only if, one being an antecedent solution of a solving triple $\left(M_{1} ; M_{2} ; M_{3}\right)$, the other is an antecedent solution of the solving triple $\left(M_{1} ; M_{2} ;-M_{3}\right)$. If we consider two such solutions, $\left(V_{1} ; V_{2} ; V_{3}\right)$ and $\left(V_{1}^{\prime} ; V_{2}^{\prime} ; V_{3}^{\prime}\right)$, then $V_{1}$ and $V_{1}^{\prime}$ are two eigenvectors of the matrix $M_{3} M_{2} M_{1}$ associated with the eigenvalues 1 and -1 , respectively. We shall use this remark in $\S 4$.

Theorem 3. The link matrices are matrices of the form $\frac{1}{Z}\left(\begin{array}{c}X-Y \\ Y \\ X-Y\end{array}\right)$, with $X=s^{2}+(s+t)^{2}, Y=t^{2}+(s+t)^{2}$, and $Z=s^{2}+3 s t+t^{2}$, where $(s ; t)$ is an arbitrary nonzero pair of rational numbers.

Proof. Every matrix $M$ of the type given in Theorem 3 is of the form $\left(\begin{array}{cc}a-b & a \\ b & a-b\end{array}\right)$, with $a=\frac{X}{Z}$ and $b=\frac{Y}{Z}$; it is easily verified that its determinant is -1 . So $M$ is a link matrix.

Conversely, let $M$ be a link matrix. We have $M=\left(\begin{array}{cc}a-b & a \\ b & a-b\end{array}\right)$, and the determinant of $M$ is -1 , so that $a^{2}-3 a b+b^{2}=-1$. Consider the following system in $s$ and $t$ :

$$
\begin{aligned}
& (a-b-1) s+(2 a-b) t=0, \\
& (a-2 b) s+(a-b+1) t=0 .
\end{aligned}
$$

Its determinant, equal to $-a^{2}+3 a b-b^{2}-1$, is zero. Hence this system has nonzero solutions in rational numbers. Let $(s ; t)$ be one of them. Put $\omega=(1+\sqrt{5}) / 2$ and $\bar{\omega}=(1-\sqrt{5}) / 2$. Then $(\omega ; \bar{\omega})$ is a basis of the $\mathbb{Q}$-vector space $\mathbb{Q}[\sqrt{5}]$, so that the preceding system is equivalent to the equality

$$
[(a-b-1) s+(2 a-b) t] \omega+[(a-2 b) s+(a-b+1) t] \bar{\omega}=0 .
$$

We may write this in the form $(a \omega+b \bar{\omega})(t \omega-s \bar{\omega})=s \omega-t \bar{\omega}$. Now $(s ; t) \neq$ $(0 ; 0)$, hence $t \omega-s \bar{\omega} \neq 0$, and $s \omega-t \bar{\omega} \neq 0$. From this we deduce

$$
\begin{aligned}
a \omega+b \bar{\omega} & =\frac{s \omega-t \bar{\omega}}{t \omega-s \bar{\omega}}=\frac{(s \omega-t \bar{\omega})^{2}}{(t \omega-s \bar{\omega})(s \omega-t \bar{\omega})} \\
& =\frac{\left(2 s^{2}+2 s t+t^{2}\right) \omega+\left(s^{2}+2 s t+2 t^{2}\right) \bar{\omega}}{s^{2}+3 s t+t^{2}}
\end{aligned}
$$

hence

$$
a=\frac{s^{2}+(s+t)^{2}}{s^{2}+3 s t+t^{2}} \quad \text { and } \quad b=\frac{t^{2}+(s+t)^{2}}{s^{2}+3 s t+t^{2}}
$$


Remark. We can give $s$ and $t$ explicitly in terms of $a$ and $b$. Indeed, the numbers $a-b-1$ and $a-b+1$ cannot both be zero. If, for instance, $a-b-1 \neq$ 0 , the system in $s$ and $t$ is equivalent to the equation $(a-b-1) s+(2 a-b) t$ $=0$, whose nonzero solutions $(s ; t)$ are given by $s=K(-2 a+b)$ and $t=$ $K(a-b-1)$, with $K \in \mathbb{Q}^{*}$.

From Theorems 2 and 3, we deduce the following consequence. Let $M_{i}$, with $1 \leq i \leq 3$, be three link matrices of the type given in Theorem 3,

$$
M_{i}=\frac{1}{Z_{i}}\left(\begin{array}{cc}
X_{i}-Y_{i} & X_{i} \\
Y_{i} & X_{i}-Y_{i}
\end{array}\right) .
$$

Then $\left(M_{1} ; M_{2} ; M_{3}\right)$ is a solving triple if and only if the trace of the matrix $M_{3} M_{2} M_{1}$ is zero, hence, from the proof of Theorem 2, if and only if $X_{1} X_{2} X_{3}=$ $Y_{1} Y_{2} Y_{3}$; this may be written as

$$
\begin{aligned}
\left(s_{1}^{2}+\right. & \left.\left(s_{1}+t_{1}\right)^{2}\right)\left(s_{2}^{2}+\left(s_{2}+t_{2}\right)^{2}\right)\left(s_{3}^{2}+\left(s_{3}+t_{3}\right)^{2}\right) \\
& =\left(t_{1}^{2}+\left(s_{1}+t_{1}\right)^{2}\right)\left(t_{2}^{2}+\left(s_{2}+t_{2}\right)^{2}\right)\left(t_{3}^{2}+\left(s_{3}+t_{3}\right)^{2}\right) .
\end{aligned}
$$

We call this last equality the condition of closure.

\section{Solutions of the Diophantine equation}

Using the condition of closure, we now give solutions of system (II). First we need matrices slightly simpler than link matrices.

Definition. We call every matrix $L$ of the form $L=\left(\begin{array}{c}X-Y \\ Y \\ X-Y\end{array}\right)$, with $X=$ $s^{2}+(s+t)^{2}, Y=t^{2}+(s+t)^{2}, s$ and $t$ rational numbers, $(s ; t) \neq(0 ; 0)$ a pre-link matrix. We shall adopt the abbreviated notation $L=\left(\begin{array}{ll}\bullet & X \\ Y & \bullet\end{array}\right)$.

1st solution. The simplest solution consists in looking for triples of pre-link matrices of the form

$$
L_{3} L_{2} L_{1}=\left(\begin{array}{cc}
\bullet & B \\
A & \bullet
\end{array}\right)\left(\begin{array}{cc}
\bullet & C \\
B & \bullet
\end{array}\right)\left(\begin{array}{cc}
\bullet & A \\
C & \bullet
\end{array}\right) .
$$

We may choose, for instance,

$$
\begin{gathered}
L_{3}=\left(\begin{array}{cc}
\bullet & (s+t)^{2}+s^{2} \\
s^{2}+t^{2} & \bullet
\end{array}\right), \quad L_{2}=\left(\begin{array}{cc}
\bullet & (s+t)^{2}+t^{2} \\
(s+t)^{2}+s^{2} & \bullet
\end{array}\right), \\
L_{1}=\left(\begin{array}{cc}
\bullet \\
(s+t)^{2}+t^{2} & s^{2}+t^{2}
\end{array}\right), \quad(s, t) \in \mathbb{Z}^{2} \backslash\{(0 ; 0)\} .
\end{gathered}
$$

The matrices $L_{i}$, for $1 \leq i \leq 3$, are obviously pre-link matrices, and the condition of closure is satisfied. From this we deduce a solving triple,

$$
\begin{aligned}
& M_{1}=\frac{1}{-s^{2}-s t+t^{2}}\left(\begin{array}{cc}
-2 s t-t^{2} & s^{2}+t^{2} \\
(s+t)^{2}+t^{2} & -2 s t-t^{2}
\end{array}\right), \\
& M_{2}=\frac{1}{s^{2}+3 s t+t^{2}}\left(\begin{array}{cc}
t^{2}-s^{2} & (s+t)^{2}+t^{2} \\
(s+t)^{2}+s^{2} & t^{2}-s^{2}
\end{array}\right), \\
& M_{3}=\frac{1}{s^{2}-s t-t^{2}}\left(\begin{array}{cc}
s^{2}+2 s t & (s+t)^{2}+s^{2} \\
s^{2}+t^{2} & s^{2}+2 s t
\end{array}\right) .
\end{aligned}
$$

We find that

$$
M_{3} M_{2} M_{1}=\frac{1}{D}\left(\begin{array}{ll}
a_{11} & a_{12} \\
a_{21} & a_{22}
\end{array}\right)
$$


with

$$
\begin{array}{llllllll}
D & =-s^{6} & -3 s^{5} t & +2 s^{4} t^{2} & +9 s^{3} t^{3} & +2 s^{2} t^{4} & -3 s t^{5} & -t^{6}, \\
a_{11}=-s^{6} & -6 s^{5} t & -6 s^{4} t^{2} & & +6 s^{2} t^{4} & +6 s t^{5} & +t^{6}, \\
a_{12}=3 s^{6} & +8 s^{5} t & +9 s^{4} t^{2} & -4 s^{3} t^{3} & -9 s^{2} t^{4} & -2 s t^{5}, & \\
a_{21}= & -2 s^{5} t & -9 s^{4} t^{2} & -4 s^{3} t^{3} & +9 s^{2} t^{4} & +8 s t^{5} & +3 t^{6}, \\
a_{22}=-a_{11} . & & & & & &
\end{array}
$$

The eigenvalue 1 of the matrix $M_{3} M_{2} M_{1}$ leads to the antecedent solution $(s ; t ;-(s+t): t ;-(s+t) ; s)$, which is trivial and nonzero.

In the case of the eigenvalue -1 , write $I$ for the identity matrix and put $P(s ; t)=3 s^{5}+8 s^{4} t+9 s^{3} t^{2}-4 s^{2} t^{3}-9 s t^{4}-2 t^{5}$. We find that

$$
M_{3} M_{2} M_{1}+I=\frac{1}{D}\left(\begin{array}{ll}
s P(t ; s) & s P(s ; t) \\
t P(t ; s) & t P(s ; t)
\end{array}\right) .
$$

Therefore, an eigenvector of $M_{3} M_{2} M_{1}$ associated with the eigenvalue -1 is $V_{1}=\left(\begin{array}{l}x_{1} \\ y_{1}\end{array}\right)$, with $x_{1}=P(s ; t)$ and $y_{1}=-P(t ; s)$. The vectors $V_{2}$ and $V_{3}$ are calculated by $V_{2}=M_{1} V_{1}$ and $V_{3}=M_{2} V_{2}$. We then find

$\mathbf{S}(5 ; 1)$

$$
\begin{aligned}
& x_{1}=3 s^{5}+8 s^{4} t+9 s^{3} t^{2}-4 s^{2} t^{3}-9 s t^{4}-2 t^{5}, \\
& x_{2}=-2 s^{5}-s^{4} t+12 s^{3} t^{2}+13 s^{2} t^{3}+4 s t^{4}-t^{5}, \\
& x_{3}=-s^{5}-9 s^{4} t-13 s^{3} t^{2}-7 s^{2} t^{3}-7 s t^{4}-3 t^{5}, \\
& y_{1}=2 s^{5}+9 s^{4} t+4 s^{3} t^{2}-9 s^{2} t^{3}-8 s t^{4}-3 t^{5}, \\
& y_{2}=-3 s^{5}-7 s^{4} t-7 s^{3} t^{2}-13 s^{2} t^{3}-9 s t^{4}-t^{5}, \\
& y_{3}=-s^{5}+4 s^{4} t+13 s^{3} t^{2}+12 s^{2} t^{3}-s t^{4}-2 t^{5} .
\end{aligned}
$$

Remarks. 1. This solution may be characterized by the fact that it is the conjugate solution of the trivial solution $(s ; t ;-(s+t) ; t ;-(s+t) ; s)$. This explains in a simple way the remarkable property of this parametrization, noticed by $J$. H. Conway (see $[1$, p. 574]).

2. The 6 by 6 determinant of the sextuple $\left(x_{1} ; x_{2} ; x_{3} ; y_{1} ; y_{2} ; y_{3}\right)$ in the basis $\left(s^{5} ; s^{4} t ; s^{3} t^{2} ; s^{2} t^{3} ; s t^{4} ; t^{5}\right)$ is nonzero. So, contrary to the case of the solutions of systems $\left(B_{1}\right)$ and $\left(B_{2}\right)$, the polynomial functions $x_{1}, x_{2}, x_{3}$, $y_{1}, y_{2}$, and $y_{3}$ are linearly independent.

2nd solution. We look for triples of pre-link matrices of the form

$$
L_{3} L_{2} L_{1}=\left(\begin{array}{cc}
\bullet & B \\
A & \bullet
\end{array}\right)\left(\begin{array}{cc}
\bullet & D \\
C & \bullet
\end{array}\right)\left(\begin{array}{cc}
\bullet & A C \\
B D & \bullet
\end{array}\right) .
$$

Thus the condition of closure is satisfied.

Put

$$
L_{3}=\left(\begin{array}{cc}
\bullet & s^{2}+(s+t)^{2} \\
t^{2}+(s+t)^{2} & \bullet
\end{array}\right)
$$

and

$$
L_{2}=\left(\begin{array}{cc}
\bullet & u^{2}+(u+v)^{2} \\
v^{2}+(u+v)^{2} & \bullet
\end{array}\right) .
$$

We have

$$
X_{1}=A C=\left(t^{2}+(s+t)^{2}\right)\left(v^{2}+(u+v)^{2}\right)=(s u+s v+t u+2 t v)^{2}+(s v-t u)^{2}
$$


and

$$
Y_{1}=B D=\left(s^{2}+(s+t)^{2}\right)\left(u^{2}+(u+v)^{2}\right)=(2 s u+s v+t u+t v)^{2}+(s v-t u)^{2} .
$$

For $L_{1}$ to be a pre-link matrix, it is sufficient that

$$
(s u+s v+t u+2 t v)+(2 s u+s v+t u+t v)=(s v-t u)
$$

This may be written as $(3 s+3 t) u=-(s+3 t) v$. Therefore, we may choose $u=s+3 t$ and $v=-(3 s+3 t)$. From this we deduce a solving triple,

$$
\begin{aligned}
& M_{3}=\frac{1}{Z_{3}}\left(\begin{array}{cc}
\bullet & s^{2}+(s+t)^{2} \\
t^{2}+(s+t)^{2} & \bullet
\end{array}\right), \quad \text { with } Z_{3}=-\left(s^{2}+3 s t+t^{2}\right), \\
& M_{2}=\frac{1}{Z_{2}}\left(\begin{array}{cc}
\bullet \\
(3 s+3 t)^{2}+(2 s)^{2} & \left.(s+3 t)^{2}+(2 s)^{2}\right), \\
M_{1}=\frac{1}{Z_{1}}\left(\left(2 s^{2}+5 s t+3 t^{2}\right)^{2}+\left(3 s^{2}+4 s t+3 t^{2}\right)^{2}\right. & \text { with } Z_{2}=-s^{2}+12 s t+9 t^{2}, \\
\text { with } Z_{1}=11 s^{4}+27 s^{3} t+32 s^{2} t^{2}+21 s t^{3}+9 t^{4}
\end{array}\right.
\end{aligned}
$$

From the eigenvalue 1, we obtain the parametric solution

$\mathrm{S}(4 ; 4 ; \mathrm{a})$

$$
\begin{aligned}
& x_{1}=3 s^{4}+9 s^{3} t+18 s^{2} t^{2}+21 s t^{3}+9 t^{4}, \\
& x_{2}=2 s^{4}+4 s^{3} t-5 s^{2} t^{2}-12 s t^{3}-9 t^{4}, \\
& x_{3}=-s^{4}-10 s^{3} t-17 s^{2} t^{2}-12 s t^{3}, \\
& y_{1}=s^{4}-3 s^{3} t-14 s^{2} t^{2}-15 s t^{3}-9 t^{4}, \\
& y_{2}=3 s^{4}+8 s^{3} t+9 s^{2} t^{2}, \\
& y_{3}=2 s^{4}+12 s^{3} t+19 s^{2} t^{2}+18 s t^{3}+9 t^{4} .
\end{aligned}
$$

Apart from exchanging $x_{1}$ and $x_{2}$, this is Brudno's solution [4] of $\left(\mathbf{B}_{2}\right)$. From the eigenvalue -1 , we obtain

$$
\begin{aligned}
& x_{1}=67 s^{4}+165 s^{3} t+178 s^{2} t^{2}+81 s t^{3}+9 t^{4}, \\
& x_{2}=36 s^{4}+140 s^{3} t+189 s^{2} t^{2}+108 s t^{3}+27 t^{4}, \\
& x_{3}=37 s^{4}+42 s^{3} t-13 s^{2} t^{2}-48 s t^{3}-18 t^{4}, \\
& y_{1}=15 s^{4}-9 s^{3} t-18 s^{2} t^{2}+3 s t^{3}+9 t^{4}, \\
& y_{2}=65 s^{4}+152 s^{3} t+169 s^{2} t^{2}+96 s t^{3}+18 t^{4}, \\
& y_{3}=-52 s^{4}-152 s^{3} t-167 s^{2} t^{2}-102 s t^{3}-27 t^{4} .
\end{aligned}
$$

Apart from multiplicative coefficients, permutations, and changes of signs, this solution is deduced from the preceding one by the transformation $s^{\prime}=2 s+3 t$, $t^{\prime}=-3 s-2 t$, which realizes a one-to-one mapping from $\mathbb{Q}^{2}$ onto $\mathbb{Q}^{2}$.

Remark. Every solution of the system

$$
\left\{\begin{array}{l}
x_{1}^{6}+x_{2}^{6}+x_{3}^{6}=y_{1}^{6}+y_{2}^{6}+y_{3}^{6}, \\
x_{1}^{2}+x_{2}^{2}+x_{3}^{2}=y_{1}^{2}+y_{2}^{2}+y_{3}^{2}, \\
x_{1}+3 x_{2}+x_{3}=3 y_{1}+y_{2}+y_{3}
\end{array}\right.
$$


(that is, $\left(\mathbf{B}_{2}\right)$, apart from exchanging $x_{1}$ and $x_{2}$ ) is a solution of (II), therefore, a fortiori, of (I). In particular, it satisfies the additional equality

$$
x_{1} y_{1}\left(x_{1}^{2}-y_{1}^{2}\right)+x_{2} y_{2}\left(x_{2}^{2}-y_{2}^{2}\right)+x_{3} y_{3}\left(x_{3}^{2}-y_{3}^{2}\right)=0 .
$$

3rd and 4th solutions. We look for pre-link matrices of the form

$$
L_{3} L_{2} L_{1}=\left(\begin{array}{cc}
\bullet & B \\
A & \bullet
\end{array}\right)\left(\begin{array}{cc}
\bullet & C \\
B X & \bullet
\end{array}\right)\left(\begin{array}{cc}
\bullet & A X \\
C & \bullet
\end{array}\right) .
$$

The condition of closure is automatically satisfied. Put

$$
L_{3}=\left(\begin{array}{cc}
\bullet & (s+t)^{2}+t^{2} \\
s^{2}+t^{2} & \bullet
\end{array}\right)
$$

and $X=u^{2}+v^{2}$. We have

$$
B X=\left((s+t)^{2}+t^{2}\right)\left(u^{2}+v^{2}\right)=(u s+u t+v t)^{2}+(v s+v t-u t)^{2} .
$$

Put $u s+u t+v t=b_{1}$ and $v s+v t-u t=b_{2}$. Thus we have $B X=b_{1}^{2}+b_{2}^{2}$. For $L_{2}$ to be a pre-link matrix, it is sufficient that $C=b_{1}^{2}+\left(b_{1}+b_{2}\right)^{2}$, that is, $C=(u s+u t+v t)^{2}+(u s+v s+2 v t)^{2}$. In the same way,

$$
A X=\left(s^{2}+t^{2}\right)\left(u^{2}+v^{2}\right)=(u s+v t)^{2}+(v s-u t)^{2} .
$$

Put $u s+v t=a_{1}$ and $v s-u t=a_{2}$. We have $A X=a_{1}^{2}+a_{2}^{2}$. For $L_{1}$ to be a pre-link matrix, it is sufficient that $C=a_{1}^{2}+\left(a_{1}-a_{2}\right)^{2}$, that is, $C=$ $(u s+v t)^{2}+(u s+u t-v s+v t)^{2}$.

By equating the two expressions of $C$, we obtain, after cancelling,

$$
v^{2}\left(6 s t+3 t^{2}\right)=u v\left(-4 s^{2}-4 s t\right) \text {. }
$$

If $v=0$, we recover our first parametric solution. If $v \neq 0$, we may choose $u=6 s t+3 t^{2}$ and $v=-4 s^{2}-4 s t$. From this we deduce $L_{1}, L_{2}$, and $L_{3}$, then $M_{1}, M_{2}$, and $M_{3}$. The two eigenvalues 1 and -1 of the matrix $M_{3} M_{2} M_{1}$ lead to the following two parametric solutions:

$$
\begin{aligned}
& x_{1}=16 s^{5}+64 s^{4} t+104 s^{3} t^{2}+116 s^{2} t^{3}+78 s t^{4}+27 t^{5}, \\
& x_{2}=16 s^{5}+24 s^{4} t+48 s^{3} t^{2}+52 s^{2} t^{3}+57 s t^{4}+18 t^{5}, \\
& x_{3}=16 s^{5}+32 s^{4} t+52 s^{3} t^{2}+48 s^{2} t^{3}+21 s t^{4}-9 t^{5}, \\
& y_{1}=16 s^{5}+48 s^{4} t+84 s^{3} t^{2}+76 s^{2} t^{3}+33 s t^{4}+18 t^{5}, \\
& y_{2}=16 s^{5}+56 s^{4} t+112 s^{3} t^{2}+108 s^{2} t^{3}+81 s t^{4}+27 t^{5}, \\
& y_{3}=16 s^{5}+16 s^{4} t+8 s^{3} t^{2}-28 s^{2} t^{3}-18 s t^{4}-9 t^{5}
\end{aligned}
$$

and

$$
\begin{aligned}
& x_{1}=[16 ; 64 ; 160 ; 308 ; 416 ; 397 ; 262 ; 139 ; 54 ; 9], \\
& x_{2}=[-16 ;-56 ;-80 ;-12 ; 119 ; 234 ; 233 ; 126 ; 27 ; 0], \\
& x_{3}=[16 ; 96 ; 236 ; 368 ; 387 ; 261 ; 75 ;-41 ;-39 ;-9], \\
& y_{1}=[-16 ;-48 ;-44 ; 60 ; 225 ; 290 ; 231 ; 106 ; 21 ; 0], \\
& y_{2}=[16 ; 88 ; 208 ; 324 ; 369 ; 355 ; 281 ; 149 ; 51 ; 9], \\
& y_{3}=[-16 ;-80 ;-224 ;-364 ;-360 ;-199 ;-34 ; 51 ; 42 ; 9] .
\end{aligned}
$$


In this last case, we write $\left[a_{0} ; \ldots ; a_{9}\right]$ to denote the polynomial, homogeneous in $s$ and $t, a_{0} s^{9}+\cdots+a_{9} t^{9}$.

5th and 6th solutions. As in the preceding case, we look for triples of pre-link matrices of the form

$$
L_{3} L_{2} L_{1}=\left(\begin{array}{cc}
\bullet & B \\
A & \bullet
\end{array}\right)\left(\begin{array}{cc}
\bullet & C \\
B X & \bullet
\end{array}\right)\left(\begin{array}{cc}
\bullet & A X \\
C & \bullet
\end{array}\right) .
$$

Put, this time,

$$
L_{3}=\left(\begin{array}{cc}
\bullet & (u+v)^{2}+v^{2} \\
u^{2}+v^{2} & \bullet
\end{array}\right)
$$

and $X=s^{2}+t^{2}$. We have

$$
B X=\left(\left(u+v^{2}\right)+v^{2}\right)\left(s^{2}+t^{2}\right)=(s u+s v+t v)^{2}+(s v-t u-t v)^{2} .
$$

Put $s u+s v+t v=b_{1}$ and $s v-t u-t v=b_{2}$. We have $B X=b_{1}^{2}+b_{2}^{2}$. For $L_{2}$ to be a pre-link matrix, it is sufficient that $C=\left(b_{1}+b_{2}\right)^{2}+b_{2}^{2}$, that is, $C=((s-t) u+2 s v)^{2}+(-t u+(s-t) v)^{2}$. In the same way,

$$
A X=\left(u^{2}+v^{2}\right)\left(s^{2}+t^{2}\right)=(s u+t v)^{2}+(s v-t u)^{2} .
$$

Put $s u+t v=a_{1}$ and $s v-t u=a_{2}$. We have $A X=a_{1}^{2}+a_{2}^{2}$. For $L_{1}$ to be a pre-link matrix, it is sufficient that $C=\left(a_{1}+a_{2}\right)^{2}+a_{2}^{2}$, that is, $C=$ $((s-t) u+(s+t) v)^{2}+(-t u+s v)^{2}$.

By equating the two expressions of $C$, we obtain $u v\left(2 s^{2}-4 s t+4 t^{2}\right)=$ $v^{2}\left(-3 s^{2}+4 s t\right)$. If $v=0$, we obtain

$$
L_{3}=\left(\begin{array}{cc}
\bullet & u^{2} \\
u^{2} & \bullet
\end{array}\right)
$$

hence $M_{3}=\left(\begin{array}{ll}0 & 1 \\ 1 & 0\end{array}\right)$, yielding a trivial solution. If $v \neq 0$, we may choose $u=-3 s^{2}+4 s t$ and $v=2 s^{2}-4 s t+4 t^{2}$. From this we deduce in succession $L_{1}, L_{2}, L_{3}, M_{1}, M_{2}, M_{3}$, then the following two parametric solutions:

$$
\begin{aligned}
& x_{1}=[15 ;-96 ; 295 ;-628 ; 920 ;-880 ; 608 ;-320 ; 64], \\
& x_{2}=[-25 ; 163 ;-483 ; 896 ;-1220 ; 1200 ;-784 ; 384 ;-128], \\
& x_{3}=[-5 ; 22 ;-84 ; 284 ;-620 ; 880 ;-816 ; 512 ;-192], \\
& y_{1}=[-25 ; 162 ;-480 ; 908 ;-1220 ; 1200 ;-816 ; 320 ;-64], \\
& y_{2}=[15 ;-109 ; 309 ;-544 ; 800 ;-960 ; 832 ;-512 ; 192], \\
& y_{3}=[-5 ;-12 ; 143 ;-376 ; 580 ;-720 ; 656 ;-384 ; 128]
\end{aligned}
$$

and

$$
\begin{aligned}
x_{1} & =[13 ;-28 ; 31 ; 68 ;-160 ; 336 ;-256 ; 192 ;-64], \\
x_{2} & =[-21 ; 55 ;-151 ; 240 ;-380 ; 368 ;-240 ; 128 ; 0], \\
x_{3} & =[1 ;-58 ; 166 ;-308 ; 460 ;-368 ; 304 ;-128 ; 64], \\
y_{1} & =[-21 ; 54 ;-154 ; 268 ;-300 ; 368 ;-272 ; 192 ;-64], \\
y_{2} & =[13 ;-39 ;-1 ; 48 ;-200 ; 256 ;-288 ; 128 ;-64], \\
y_{3} & =[-1 ;-64 ; 161 ;-320 ; 420 ;-432 ; 272 ;-128 ; 0] .
\end{aligned}
$$


7th and 8th solutions. We look for triples of pre-link matrices of the form

$$
L_{3} L_{2} L_{1}=\left(\begin{array}{cc}
\bullet & B X \\
A Y & \bullet
\end{array}\right)\left(\begin{array}{cc}
\bullet & C Y \\
B Z & \bullet
\end{array}\right)\left(\begin{array}{cc}
\bullet & A Z \\
C X & \bullet
\end{array}\right)
$$

The condition of closure is obviously satisfied. Put

$$
\begin{aligned}
& L_{3}=\left(\begin{array}{cc}
\bullet & \left((s+t)^{2}+t^{2}\right)\left(a^{2}+b^{2}\right)
\end{array}\right), \\
& L_{2}=\left(\begin{array}{cc}
\left(s^{2}+t^{2}\right)\left(c^{2}+d^{2}\right) & \bullet \\
\left(t^{2}+(s+t)^{2}\right)\left(e^{2}+f^{2}\right) & \left(s^{2}+(s+t)^{2}\right)\left(c^{2}+d^{2}\right)
\end{array}\right), \\
& L_{1}=\left(\begin{array}{cc}
\bullet & \left(s^{2}+t^{2}\right)\left(e^{2}+f^{2}\right)
\end{array}\right) .
\end{aligned}
$$

We may write

$$
\begin{gathered}
L_{3}=\left(\begin{array}{cc}
\bullet & X_{3}^{2}+Y_{3}^{2} \\
Z_{3}^{2}+T_{3}^{2} & \bullet
\end{array}\right), \quad \text { with: } \begin{array}{ll}
X_{3}=-a s-a t+b t, & Y_{3}=-b s-b t-a t, \\
Z_{3}=c s+d t, & T_{3}=d s-c t,
\end{array} \\
L_{2}=\left(\begin{array}{cc}
\bullet & X_{2}^{2}+Y_{2}^{2} \\
Z_{2}^{2}+T_{2}^{2} & \bullet
\end{array}\right), \quad \text { with: } \begin{array}{ll}
X_{2}=c s-d s-d t, & Y_{2}=d s+c s+c t \\
Z_{2}=e t-f s-f t, & T_{2}=f t+e s+e t
\end{array} \\
L_{1}=\left(\begin{array}{cc}
\bullet & X_{1}^{2}+Y_{1}^{2} \\
Z_{1}^{2}+T_{1}^{2} & \bullet
\end{array}\right), \quad \text { with: } \begin{array}{ll}
X_{1}=e t+f s, & Y_{1}=f t-e s, \\
Z_{1}=-a s-a t+b s, & T_{1}=-b s-b t-a s .
\end{array}
\end{gathered}
$$

For every matrix $L_{i}$ to be a pre-link matrix, it is sufficient that $Y_{i}=T_{i}$ and $X_{i}+Z_{i}+T_{i}=0$ (for $\left.1 \leq i \leq 3\right)$.

Now if $Y_{i}=T_{i}$ for $1 \leq i \leq 3$, we have $Y_{1}+Y_{2}+Y_{3}=T_{1}+T_{2}+T_{3}$. After cancelling, this equality is reduced to $s(a+c-2 e)=t(a-2 c+e)$. A sufficient condition is that $a+c-2 e=0$ and $a-2 c+e=0$, that is, $a=c=e$. By replacing $c$ and $e$ by $a$ in every equality $Y_{i}=T_{i}$, we obtain the equalities $-b(s+t)=d s=f t$. A simple sufficient condition is that $b=-2 s t$, $d=2 t(s+t)$, and $f=2 s(s+t)$.

Then, if we replace $c$ and $e$ by $a, b$ by $-2 s t, d$ by $2 t(s+t)$, and $f$ by $2 s(s+t)$ in the equalities $X_{i}+Z_{i}+T_{i}=0$ for $1 \leq i \leq 3$, we obtain $t\left(a-s^{2}-s t-t^{2}\right)=0,(s+t)\left(a-s^{2}-s t-t^{2}\right)=0$, and $s\left(a-s^{2}-s t-t^{2}\right)=0$.

A simple sufficient condition is that $a=s^{2}+s t+t^{2}$. There follow the parametric solutions

$$
\begin{aligned}
x_{1} & =[2 ; 7 ; 15 ; 18 ; 9 ; 2 ; 1 ; 1], \\
x_{2} & =[1 ; 1 ;-4 ;-17 ;-22 ;-15 ;-7 ;-2], \\
x_{3} & =[-1 ;-6 ;-17 ;-21 ;-17 ;-11 ;-6 ;-1], \\
y_{1} & =[-1 ;-1 ;-2 ;-9 ;-18 ;-15 ;-7 ;-2], \\
y_{2} & =[1 ; 6 ; 11 ; 17 ; 21 ; 17 ; 6 ; 1], \\
y_{3} & =[2 ; 7 ; 15 ; 22 ; 17 ; 4 ;-1 ;-1]
\end{aligned}
$$


and

$\mathrm{S}(11 ; 7)$

$$
\begin{gathered}
x_{1}=[8 ; 43 ; 167 ; 547 ; 1296 ; 1863 ; 1631 ; 927 ; 352 ; \\
63 ;-17 ;-5], \\
x_{2}=[-1 ; 39 ; 213 ; 610 ; 1005 ; 939 ; 587 ; 412 ; 341 ; \\
177 ; 45 ; 8], \\
x_{3}=[5 ; 38 ; 42 ;-155 ;-769 ;-1554 ;-1744 ;-1217 ; \\
-613 ;-232 ;-50 ;-1], \\
y_{1}=[-5 ;-17 ; 63 ; 352 ; 927 ; 1631 ; 1863 ; 1296 ; 547 ; \\
167 ; 43 ; 8], \\
y_{2}=[1 ; 50 ; 232 ; 613 ; 1217 ; 1744 ; 1554 ; 769 ; 155 ; \\
-42 ;-38 ;-5], \\
y_{3}=[-8 ;-45 ;-177 ;-341 ;-412 ;-587 ;-939 ; \\
-1005 ;-610 ;-213 ;-39 ; 1] .
\end{gathered}
$$

Remarks. 1. Consider system $\left(\mathrm{B}_{1}\right)$ that Brudno and Kaplansky [3] solved, by having recourse to the rational points on a cubic. It is easily verified that, for every solution of $\left(B_{1}\right)$,

$$
\text { if } x_{1} x_{2} x_{3} y_{1}<0 \text {, then }\left(x_{1} ; x_{2} ; x_{3} ; y_{1} ; y_{2} ;-y_{3}\right) \text { is a solution of (II) }
$$

and

if $x_{1} x_{2} x_{3} y_{1}>0$, then $\left(x_{1} ; x_{2} ; x_{3} ;-y_{1} ; y_{2} ;-y_{3}\right)$ is a solution of (II).

So it is natural to consider, for instance, the following system $\left(B_{1}-\right.$ II $)$, deduced from $\left(\mathbf{B}_{1}\right)$ by replacing $y_{3}$ by $-y_{3}$, and by adding an inequality to it:

$$
\left\{\begin{array}{l}
x_{1}^{6}+x_{2}^{6}+x_{3}^{6}=y_{1}^{6}+y_{2}^{6}+y_{3}^{6}, \\
x_{1}^{2}+x_{2}^{2}+x_{3}^{2}=y_{1}^{2}+y_{2}^{2}+y_{3}^{2}, \\
y_{2}=x_{2}-x_{3} \\
-y_{3}=x_{2}+x_{3}, \\
x_{1} x_{2} x_{3} y_{1}<0 .
\end{array}\right.
$$

From the beginning of this remark, every solution of this system is a solution of (II). More precisely, a nonzero solution of (II), with associated solving triple $\left(M_{1} ; M_{2} ; M_{3}\right)$, is a solution of $\left(\mathrm{B}_{1}-\mathrm{II}\right)$ if and only if $M_{2}=\left(\begin{array}{cc}1 & -1 \\ -2 & 1\end{array}\right)$.

2. The seventh solution of Lander, Parkin, and Selfridge [5], after exchanging $y_{1}$ and $y_{2}$, that is, put in the form $(51 ; 113 ; 136 ; 125 ; 40 ; 129)$, is a solution of (II). However, an examination of its solving triple shows that it can be obtained neither as one of Brudno's solutions of systems $\left(B_{1}\right)$ or $\left(B_{2}\right)$, nor by one of our parametric solutions.

3. Permutations and changes of signs apart, all the known solutions of the system

$$
\left\{\begin{array}{l}
x_{1}^{6}+x_{2}^{5}+x_{3}^{6}=y_{1}^{6}+y_{2}^{6}+y_{3}^{6}, \\
x_{1}^{2}+x_{2}^{2}+x_{3}^{2}=y_{1}^{2}+y_{2}^{2}+y_{3}^{2}
\end{array}\right.
$$

are solutions of (II). It is still an unsolved problem to find a counterexample.

4. In 1979, Bremner [1] studied the surface $V$ defined by (II), by using techniques of algebraic geometry. He determined a basis for the Néron-Severi 
groups of $V$ over the fields $\mathbb{Q}(i)$ and $\mathbb{Q}$, and he exhibited a system of thirteen generators over $\mathbb{Q}$. This allowed him to determine all parametric solutions of (II) of a given degree thanks to an algorithm whose results corroborate our own, sometimes, however, with difficulty.

It would be interesting to determine how our results could be interpreted in terms of the framework of [1], but this does not seem straightforward.

\section{ACKNOWLEDGMENTS}

The author thanks Mr. Simon J. Agou, professor at the Universite du Maine and at Claude Bernard University Lyon I, for his help and guidance. He also wishes to thank the referee for his useful remarks and suggestions.

\section{BIBLIOGRAPHY}

1. Andrew Bremner, A geometric approach to equal sums of sixth powers, Proc. London Math. Soc. 43 (1981), 544-581.

2. Simcha Brudno, On generating infinitely many solutions of the Diophantine equation $A^{6}+$ $B^{6}+C^{6}=D^{6}+E^{6}+F^{6}$, Math. Comp. 24 (1970), 453-454.

3. Simcha Brudno and Irving Kaplansky, Equal sums of sixth powers, J. Number Theory 6 (1974), 401-403.

4. Simcha Brudno, Triples of sixth powers with equal sums, Math. Comp. 30 (1976), 646-648.

5. L. J. Lander, T. R. Parkin, and J. L. Selfridge, A survey of equal sums of like powers, Math. Comp. 21 (1967), 446-459.

6. K. Subba-Rao, On sums of sixth powers, J. London Math. Soc. 9 (1934), 172-173.

Jean-Joël Delorme, c/o Professor Simon J. Agou, Department of Mathematics and Computer Sciences, Université du Maine, BP 535, 72017 Le Mans, France 